\title{
FUNCTIONS WHICH ARE FOURIER-STIELTJES TRANSFORMS
}

\section{STEPHEN H. FRIEDBERG}

ABstract. Let $G$ be a locally compact abelian group, $\hat{G}$ the dual group, $M(G)$ the algebra of regular bounded Borel measures on $G$, and $M(G)^{\wedge}$ the algebra of Fourier-Stieltjes transforms. The purpose of this paper is to characterize those continuous functions on $\hat{G}$ which belongs to $M(X)^{\wedge}$, where $X$ is a closed subset of $G$ and $M(X)=\{\mu \in M(G)$ : the support of $\mu$ is contained in $X\}$.

More precisely, we will prove the following theorem:

THEOREM. Let $X$ be a closed subset of $G$ and $f$ a continuous function on $\hat{G}$. Then the following are equivalent:

(a) $f \in M(X)^{\wedge}$.

(b) $\left\{\lambda_{n}\right\} \subset M(\hat{G}),\left|\hat{\lambda}_{n}(x)\right| \leqq M$ for all $x \in \dot{X}$ and $\lambda_{n}(x) \rightarrow 0$ for all $x \in X$ implies $\int_{\hat{G}} f d \lambda_{n} \rightarrow 0$.

The case where $f$ is assumed bounded and $X=G$ was proved by Ramirez in [2] by applying Grothendieck's completion theorem [1, p. 271] to the paired spaces $M(G)^{\wedge}$ and $M(\hat{G})$ under the pairing $(\hat{\mu}, \lambda)=\int_{\hat{\theta}} \hat{\mu} d \lambda$ where $\mu \in M(G)$ and $\lambda \in M(\hat{G})$.

We provide a short proof of the more general result using the wellknown theorem of Eberlein (see, for example [3, p. 32]), which states that a continuous function $f$ on $\hat{G}$ is a Fourier-Stieltjes transform if and only if there exists a constant $A$ such that

$$
\left|\sum_{i=1}^{n} c_{i} f\left(\gamma_{i}\right)\right| \leqq A\|p\|_{\infty}, \quad \gamma_{i} \in \hat{G}
$$

for every trigonometric polynomial $p$ on $G$ of the form

$$
p(x)=\sum_{i=1}^{n} c_{i} \gamma_{i}(x), \quad x \in G .
$$

Proof. Suppose $f=\hat{\mu}$ where $\mu \in M(X)$ and $\left\{\lambda_{n}\right\}$ satisfies the hypotheses of (b). Then by Fubini's theorem and the Lebesgue dominated convergence theorem, we have

Received by the editors July $10,1970$.

AMS 1969 subject classifications. Primary 4250, 4252.

Key words and phrases. Locally compact abelian group, dual group, FourierStieltjes transform, Grothendieck's completion theorem, Eberlein's theorem, Bohr compactification. 


$$
\int_{\partial} f d \lambda_{n}=\int_{X} \lambda_{n} d \mu \rightarrow 0 .
$$

Now assume that $f$ satisfies (b). We will first show that $f \in M(G)^{\wedge}$. By Eberlein's theorem we must show that if $\left\{p_{n}\right\}$ is a sequence of trigonometric polynomials on $G$, say $p_{n}(x)=\sum_{i=1}^{k(n)} c_{i n} \gamma_{i n}(x)$ where $x \in G$ and $\gamma_{i n} \in \hat{G}$, with $p_{n} \rightarrow 0$ uniformly on $G$, then $\sum_{i=1}^{k(n)} c_{i n} f\left(\gamma_{i n}\right) \rightarrow 0$ as $n \rightarrow \infty$.

Now let $\lambda_{n}=\sum_{i=1}^{k(n)} c_{i n} \delta_{i n} \in M(\hat{G})$ where $\delta_{i n}$ is the point mass at $\gamma_{i n}$. Since $\hat{\lambda}_{n}=p_{n}$ for every $n$, we have that $\left\{\lambda_{n}\right\}$ satisfies the hypotheses of (b) and hence

$$
\sum_{i=1}^{k(n)} c_{i n} f\left(\gamma_{i n}\right)=\int_{\hat{G}} f d \lambda_{n} \rightarrow 0 \quad \text { as } n \rightarrow \infty .
$$

Therefore $f=\hat{\mu}$ where $\mu \in M(G)$ and hence we need only show that the support of $\mu$ is contained in $X$. Suppose that this is not the case. Then the regularity of $\mu$ allows us to choose a compact set $E \subset X^{\prime}$, the complement of $X$, such that $\mu(E) \neq 0$ and a sequence $\left\{U_{n}\right\}$ of open sets satisfying $E \subset U_{n} \subset X^{\prime}$ and $|\mu|\left(U_{n} \backslash E\right)<n^{-1}$ for every $n$.

Now choose a sequence $\left\{\lambda_{n}\right\} \subset M(\hat{G})$ with $0 \leqq \lambda_{n} \leqq 1, \hat{\lambda}_{n}=0$ outside $U_{n}$, and $\lambda_{n}=1$ on $E$ for every $n$. Clearly $\left\{\lambda_{n}\right\}$ satisfies the hypotheses of (b) and hence $\int_{\hat{\sigma}} f d \lambda_{n} \rightarrow 0$.

However,

$$
\begin{aligned}
\left|\int_{\hat{G}} f d \lambda_{n}\right| & =\left|\int_{U_{n}} \lambda_{n} d \mu\right| \geqq\left|\int_{E} \lambda_{n} d \mu\right|-\left|\int_{U_{n} \backslash E} \lambda_{n} d \mu\right| \\
& \geqq|\mu(E)|-n^{-1} \rightarrow|\mu(E)| \neq 0 .
\end{aligned}
$$

But this is a contradiction.

REMARK. It should be noted that if the assumption of continuity is dropped and $X$ is replaced by $G$, then (b) will imply that $f \in M(\bar{G})^{\wedge}$, where $\bar{G}$ is the Bohr compactification of $G$.

\section{REFERENCES}

1. G. Köthe, Topologische lineare räume. I, Die Grundlehren der math. Wissenschaften, Band 107, Springer-Verlag, Berlin, 1960. MR 24 \#A411.

2. D. E. Ramirez, Uniform approximation by Fourier-Stieltjes transforms, Proc. Cambridge Philos. Soc. 64 (1968), 323-333. MR 36 \#4273.

3. W. Rudin, Fourier analysis on groups, Interscience Tracts in Pure and Appl. Math., no. 12, Interscience, New York, 1962. MR 27 \#2808.

ILlinois State University, NoRmal, ILLINOIS 61761 